\title{
Conduction-electron screening in the bulk and at low-index surfaces of Ta metal
}

\author{
D. M. Riffe \\ Department of Physics, Utah State University, Logan, Utah 84322-4415 \\ W. Hale, B. Kim, ${ }^{*}$ and J. L. Erskine \\ Department of Physics, University of Texas, Austin, Texas 78712
}

(Received 17 October 1994)

\begin{abstract}
High-resolution core-level photoemission spectra from $\mathrm{Ta}(100)$ have been measured. The wellresolved peak from the first atomic layer allows a separate assessment of bulk and surface-layer screening responses: singularity indices $\alpha_{B}=0.10 \pm 0.01$ and $\alpha_{100}=0.205 \pm 0.025$, respectively, are obtained. The measured surface-atom lifetime broadening of $70 \pm 20 \mathrm{meV}$ (compared to $37 \pm 5 \mathrm{meV}$ in the bulk) is consistent with published Auger-photoemission coincidence measurements. The result for the bulk singularity index has been applied in the analysis of previous data in order to extract the screening response in the first atomic layer of $\mathrm{Ta}(111)\left(\alpha_{111}=0.16 \pm 0.01\right)$ and $\mathrm{Ta}(110)\left(\alpha_{110}=0.150 \pm 0.015\right)$. The trend in surface $\alpha$ 's implies an increasingly atomic character for the surface-layer atoms as one proceeds from $\mathrm{Ta}(110)$ to $\mathrm{Ta}(111)$ to $\mathrm{Ta}(100)$.
\end{abstract}

\section{INTRODUCTION}

The low-index surfaces of $\mathrm{W}$ and $\mathrm{Ta}$ have been widely studied with core-level photoemission as paradigms for the surface electronic structure of $d$-band transition metals. Their popularity arises from the inherently narrow width of the intense $4 f_{7 / 2}$ photopeaks combined with the relatively large difference in core-electron binding energies between atoms in the bulk and those in the first atomic layer. ${ }^{1}$ Indeed, the effects of surface orientation, reconstruction, and adsorbates on $W$ and $T a$ surfaceatom core-electron binding energies have all been investigated and continue to draw interest. ${ }^{2-9}$ Recently, on W(110), the scope of these investigations was enlarged to include discrimination of separate bulk and surface line shapes: differences in conduction-electron screening [manifest as a high-binding-energy power-law tail characterized by the singularity index $\alpha$ (Ref. 10)] and Auger core-valence-valence decay rates (manifest as a Lorentzian broadening) were discerned. ${ }^{3}$ For Ta, however, surface line-shape issues are not so well delineated. A recent high-resolution study of $\mathrm{Ta}(110),{ }^{4}$ which has a surface core-level shift ${ }^{11}$ (SCS) comparable in magnitude to that on W(110), was unable to distinguish separate lifetime and singularity-index values for the surface and bulk primarily because the increased phonon broadening in $\mathrm{Ta}$ (compared to $\mathrm{W}$ ) decreased the effective resolution of the spectra.

Fortunately, the $\mathrm{Ta}(100)$ surface offers a natural solution to this reduction in resolution since its SCS is much greater than on $\mathrm{Ta}(110)$. In fact, at least two separate core-level photoemission studies of $\mathrm{Ta}(100)$ have already been performed. ${ }^{5,6}$ However, interpretation of the spectra has been inconsistent. In the first study ${ }^{5}$ separate lifetime widths and singularity indices were ascribed to the surface and bulk components, although no accommodation for phonon broadening was made. In the later study ${ }^{6}$ the surface and bulk peaks were fit with identical values of the lifetime width and singularity index, but different phonon broadening was assumed. Additionally, the surface and underlayer core-level shifts are not consistent between the two studies.

Here we present high-resolution core-level photoemission data on $\mathrm{Ta}(100)$ which resolves these line-shape issues and establishes the surface and underlayer core-level shifts (UCS's) for this surface. We find, as for W(110), that the lifetime width and singularity index are significantly enhanced in the surface layer, suggesting a general trend for $d$-band transition metals. We have further used the bulk singularity index to analyze previously published data on $\mathrm{Ta}(111)$ and $\mathrm{Ta}(110)$, and have extracted the screening response to a surface-atom core hole for these surface orientations as well. These singularityindex results for the three Ta low-index surfaces are consistent with an increasingly atomiclike character of the surface atoms as one proceeds from $\mathrm{Ta}(110)$ to $\mathrm{Ta}(111)$ to $\mathrm{Ta}(100)$, in agreement with their relative SCS's.

\section{EXPERIMENTAL DETAILS}

The core-level data were obtained on the University of Texas/Sandia beam line on the vacuum ultraviolet (vuv) ring at the National Synchrotron Light Source at Brookhaven National Laboratory. Photon energies of 70 and $100 \mathrm{eV}$ were used to collect data at a total resolution of $130 \pm 10 \mathrm{meV}$. The Ta crystal (1-cm diameter by 0.1 $\mathrm{cm}$ thick) was oriented (to within $\pm 0.5^{\circ}$ ), spark cut, and mechanically polished. The crystal was cleaned by repeated annealing in a base-pressure vacuum of $8 \times 10^{-11}$ Torr to $2850 \mathrm{~K}^{12}$ until core-level spectra exhibited no contamination features and could be well fit with Doniach-Šunjić line shapes ${ }^{10}$ for all components. Data were collected at $80 \mathrm{~K}$ in order to minimize broadening caused by phonon excitation. 


\section{RESULTS AND ANALYSIS}

A spectrum taken with a photon energy of $70 \mathrm{eV}$ encompassing the whole $4 f$ region is displayed in Fig. 1 . Within each spin-orbit manifold $\left(j=\frac{5}{2}\right.$ and $\frac{7}{2}$ ) is a pair of peaks; the one at higher binding energy is from the first atomic layer (surface) while the lower-binding-energy peak is from the second atomic layer (underlayer) and deeper. $^{5,6}$ The spectra immediately suggest that the surface features are more asymmetric, implying that the surface singularity index is indeed larger. As evident in the figure, the background is quite small over the whole $4 f$ region. From this specta we deduce a spin-orbit splitting between the $j=\frac{5}{2}$ and $\frac{7}{2}$ components of $1.92 \pm 0.01 \mathrm{eV}$, in good agreement with a previous determination of $1.915 \pm 0.003 \mathrm{eV}^{4}$

In order to extract physical quantities related to the $4 f$ spectrum, the data were nonlinear least squares analyzed. Critical examination focused upon the $4 f_{7 / 2}$ region since the $j=\frac{7}{2}$ components are inherently better resolved. ${ }^{13}$ In the analysis the line shape of each component is described by a Doniach-Šunjić (DS) function which combines a Lorentzian width $\Gamma$ with the singularity index $\alpha{ }^{10}$ A Gaussian contribution $G$ to the line shape which includes phonon, instrumental, and any possible inhomogeneous ${ }^{14}$ broadening is introduced by convolution. In all analyses of $\mathrm{Ta}(100)$ spectra the underlayer line shape is constrained to be the same as the bulk. No constraint was place upon the underlayer-component intensity since photoelectron diffraction in Ta strongly alters component intensities away from a simple escape-depth description. ${ }^{15}$ Over the $4 f_{7 / 2}$ region of the spectrum the background is adequately described by a linear function.

A $4 f_{7 / 2}$ spectrum taken with $100-\mathrm{eV}$ photon energy and its associated nonlinear least-squares fit is illustrated in Fig. 2. The spectrum consists of a surface component due to the first atomic layer, an underlayer component from the second atomic layer, and a bulk component from atoms further away from the surface. ${ }^{5}$ The residuals, which are statistical in nature, indicate the high qual-

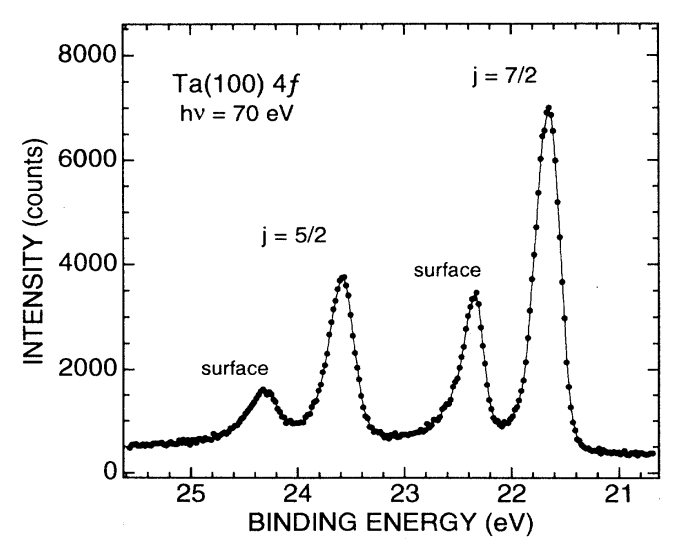

FIG. 1. Ta(100) $4 f$ photoemission spectra including the $j=\frac{5}{2}$ (higher binding energy) and $j=\frac{7}{2}$ (lower binding energy) components taken at $80 \mathrm{~K}$ with $70-\mathrm{eV}$ photon energy.

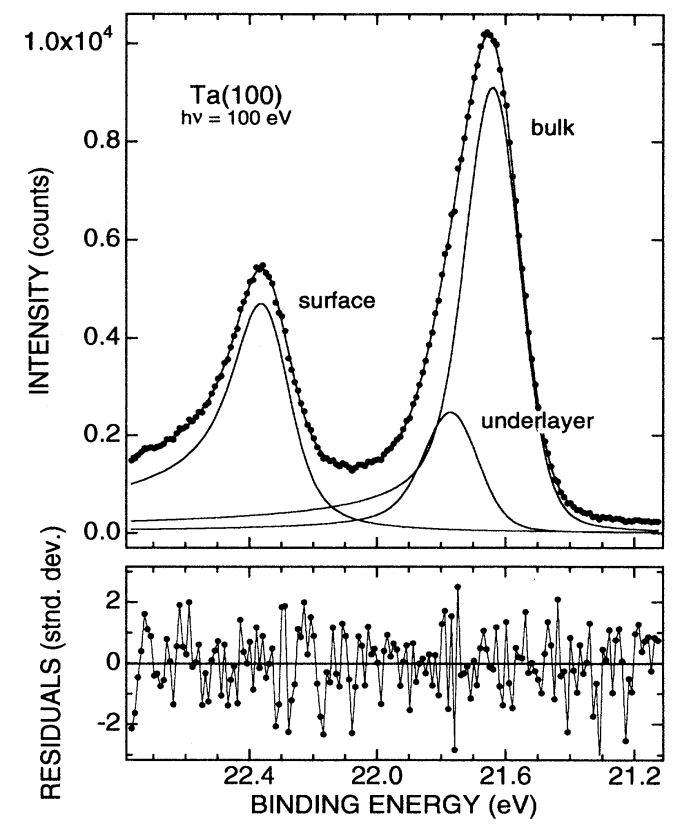

FIG. 2. Ta $4 f_{7 / 2}$ spectra taken at $80 \mathrm{~K}$ with $100-\mathrm{eV}$ photon energy. The solid line through the data is a fit made up of the three labeled components (bulk, underlayer, and surface) and a linear background (not shown). The difference between the data and the fit is shown as residuals.

ity of the fit. As suggested from the $4 f$ spectra in Fig. 1, the surface line is significantly more asymmetric (larger singularity index $\alpha$ ) than the bulk component. A larger Lorentzian (lifetime) width, which is responsible for the low-binding-energy tails of the components, is also apparent for the surface atoms.

Quantitative results for the bulk and surface line shapes from analysis of $4 f_{7 / 2}$ spectra collected at 70 and $100 \mathrm{eV}$ were consistent, and are summarized in Table I. The most distinguishing feature between the bulk and surface atoms is the difference in singularity indices, $\alpha_{B}=0.10 \pm 0.01$ and $\alpha_{100}=0.205 \pm 0.025$. The lifetime width for the surface is also larger, $70 \pm 20 \mathrm{meV}$ compared to $37 \pm 5 \mathrm{meV}$. The much larger uncertainty in the surface width arises because the low-binding-energy tail of the surface component is buried under the DS power-law tails of the bulk and underlayer components. Somewhat surprisingly, the $80-\mathrm{K}$ Gaussian broadening is smaller at the surface than in the bulk.

Using our result for the bulk singularity index, $\alpha_{B}=0.10 \pm 0.01$, we have reanalyzed previously published data from $\mathrm{Ta}(111)$ (Refs. 7 and 8) and $\mathrm{Ta}(110)$ (Ref.

TABLE I. Line-shape parameter for $\mathrm{Ta}(100) 4 f_{7 / 2}$ core levels.

\begin{tabular}{lcc}
\hline \hline Parameter & Bulk & Surface \\
\hline$\Gamma(\mathrm{meV})$ & $37 \pm 5$ & $70 \pm 20$ \\
$\alpha$ & $0.10 \pm 0.01$ & $0.205 \pm 0.025$ \\
$G(\mathrm{meV})$ & $173 \pm 5$ & $154 \pm 15$ \\
\hline \hline
\end{tabular}




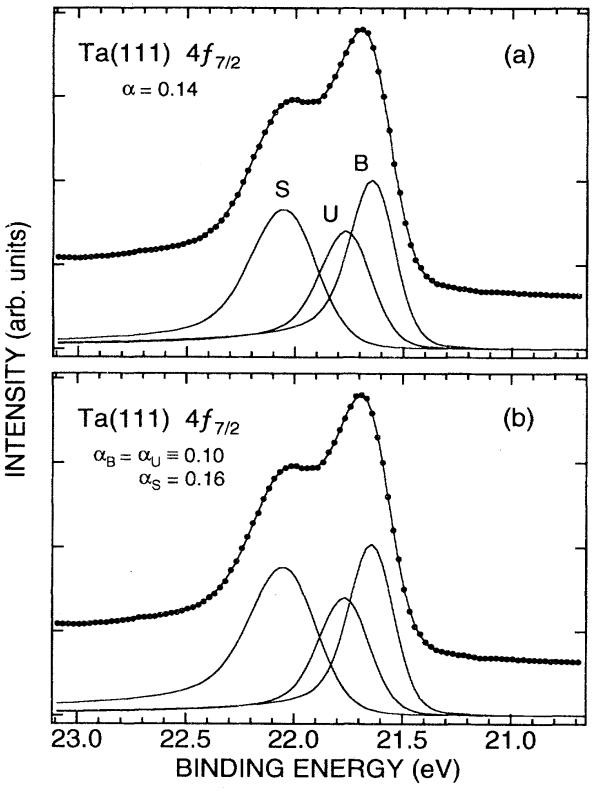

FIG. 3. Ta(111) data from Refs. 7 and 8. In (a) a fit is shown with $\alpha$ constrained to be the same for all components ( $B$, bulk; $S$, surface; and $U$, underlayer). In the fit shown in (b) $\alpha_{B}$ and $\alpha_{U}$ are constrained to 0.10 .

4) in order to determine singularity indices associated with atoms at these surfaces. Two analyses of the Ta(111) data are shown in Fig. 3. In Fig. 3(a) we first show a fit using the model function of Ref. 8, where $\alpha$ is constrained to be the same for all three componentssurface, underlayer, and bulk. From this we extract $\alpha=0.14 \pm 0.01$. In Fig. 3(b) we show a fit where the bulk and underlayer exponents $\alpha_{B}$ and $\alpha_{U}$ are constrained to 0.10 . This results in a significant increase of $\alpha_{111}$ to $0.16 \pm 0.01$. The quality of the fit is unchanged. Data taken in a previous $\mathrm{Ta}(110)$ study $^{4}$ have also been reanalyzed. Fits analogous to those in Fig. 3 for Ta(111) are illustrated for a $\mathrm{Ta}(110)$ data set in Fig. 4. For $\alpha_{B}=\alpha_{U}=0.10 \pm 0.01$ we obtain $\alpha_{110}=0.150 \pm 0.015$ from analysis of all data from the previous study. ${ }^{4}$ These fits, with $\alpha_{B}=0.10 \pm 0.01$, also slightly alter the deduced SCS and UCS values obtained from previous analyses. ${ }^{4,8} \mathrm{We}$ present the results for the surface singularity indices, SCS's, and UCS's derived from our analyses in Table II.

\section{DISCUSSION}

Before discussing the implications of the line-shape results for the three Ta surfaces, let us recall the standard model of SCS's for $d$-band transition metals. ${ }^{16}$ In this model the SCS's are related to the degree of atomic (vs metallic bonding) character the conduction $d$ band has in the vicinity of a particular atom. Compared to an atom in the bulk, an atom at the surface has a charge density which is more atomiclike because it has fewer bonds with neighboring atoms. The smaller degree of bonding leads to a narrowing of the $d$ band about its centroid which leads to a small charge flow in order to keep the surface

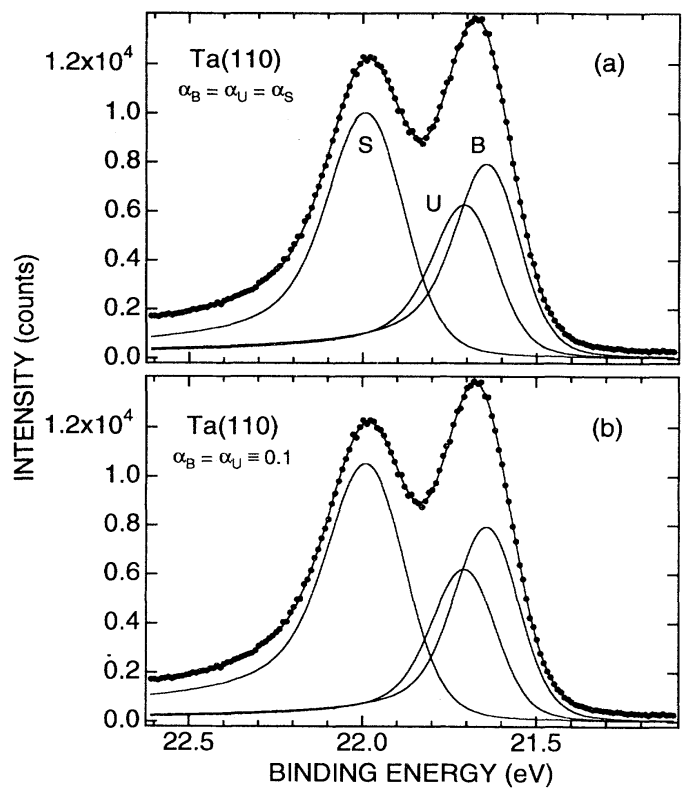

FIG. 4. Same as Fig. 3 except for Ta(110) data from Ref. 4.

and bulk atoms at the same chemical potential. For Ta this charge flow results in positive SCS's because the $d$ band is less than half filled. ${ }^{16}$ Based on their SCS's one would conclude, within the context of this model, that the electronic structure of $\mathrm{Ta}(100)$ surface atoms is the most atomiclike of the three low-index surfaces, that of $\mathrm{Ta}(110)$ is the least atomiclike, and that of $\mathrm{Ta}(111)$ falls in between.

The trend in surface-atom singularity-index values is consistent with this ordering of relative atomic character. Another characteristic of the transition between the bulk-metallic state and the atomic state for $\mathrm{Ta}$ is the amount of $s$ and $d$ charge in the valence band. For atomic Ta the electronic structure is $5 d^{3} 6 s^{2}$; the bulk-metallic state is best described as $5 d^{4} 6 s^{1}$. Atoms at the surface necessarily have an electronic structure somewhere in between. As we now show, this is manifest in the singularity index $\alpha$.

The singularity index can be described in terms of the orbital character of the conduction-band charge which screens the (photoemission final-state) core hole. In terms of the partial screening charge $q_{l}$, i.e., the amount of charge screening the core hole with orbital character $l$, the singularity index can be written as ${ }^{17}$

$$
\alpha=\sum_{l} \frac{q_{l}^{2}}{2(2 l+1)} \text {. }
$$

TABLE II. Surface singularity indices, surface core-level shifts, and underlayer core-level shifts for $\mathrm{Ta}(100), \mathrm{Ta}(111)$, and $\mathrm{Ta}(110)$.

\begin{tabular}{lccc}
\hline \hline Surface & $\alpha$ & SCS (meV) & UCS (meV) \\
\hline $\mathrm{Ta}(100)$ & $0.205 \pm 0.025$ & $718 \pm 5$ & $125 \pm 5$ \\
$\mathrm{Ta}(111)$ & $0.16 \pm 0.01$ & $390 \pm 10$ & $127 \pm 7$ \\
$\mathrm{Ta}(110)$ & $0.150 \pm 0.015$ & $350 \pm 12$ & $65 \pm 15$ \\
\hline \hline
\end{tabular}


From Eq. (1) it is readily apparent that $\alpha$ tends to decrease as the orbital angular momentum of the screening charge increases. The $l$ th partial screening charge can be written in terms of the Friedel phase shift $\delta_{l}$ as $q_{l}=(2 / \pi)(2 l+1) \delta_{l}$. Since the core hole has unit positive charge, the Friedel sum rule ${ }^{18}$ becomes simply

$$
1=\sum_{l} q_{l}
$$

The sum rule as written in Eq. (2) thus provides a constraint on the values of the contributing $q_{l}$ 's for a given value of $\alpha$.

For $\mathrm{Ta}$, whose conduction band is constructed out of atomic $s$ and $d$ orbitals, we expect the screening charge to largely reside in $s, p$, and $d$ channels, i.e, the $l=0,1$, and 2 channels, and to be negligible in the higher-l orbitals. This is supported by a band-structure calculation of the $Z+1$ metal, $W$, which shows that the charge at the Fermi level has only $3 \% l=3$ character. ${ }^{19}$ Therefore for Ta we consider only the three lowest angular-momentum channels in the screening process. In this case Eqs. (1) and (2) can be combined to yield

$$
\alpha=\frac{1}{2} q_{0}^{2}+\frac{1}{6} q_{1}^{2}+\frac{1}{10}\left(1-q_{0}-q_{1}\right)^{2} .
$$

In Fig. 5 we plot $\alpha$ vs $q_{0}$ for several fixed values of $q_{1}$. Interestingly, for $q_{0}>0.3$ the value of $\alpha$ is largely insensitive to $q_{1}$ for values of $q_{1}<0.4$. Hence, approximately, $\alpha$ depends only on $q_{0}$ for $q_{0}>0.3$ and $q_{1}<0.4$. The horizontal bars in Fig. 5 vertically mark the experimental values of the Ta bulk and low-index-surface singularity indices. The horizontal event of each bar spans the $q_{0}$ region which is consistent with $0<q_{1}<0.4$ and the uncertainty in each experimental $\alpha$. Values of $q_{1}>0.4$ appear

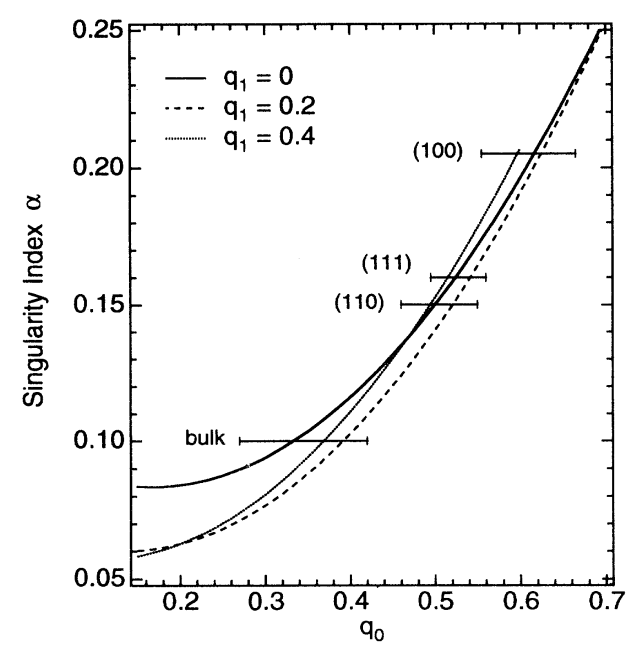

FIG. 5. Singularity index $\alpha$ vs $q_{0}$ for three fixed values of $q_{1}$ $\left(q_{1}=0\right.$, solid line; $q_{1}=0.2$, dashed line; and $q_{1}=0.4$, dotted line). The horizontal lines labeled bulk, (110), (111), and (100) vertically mark experimental values of the bulk and respective surface $\alpha$ 's for Ta metal. The horizontal extent of each bar marks the $q_{0}$ region consistent with $q_{1}<0.4$ and the experimental uncertainty in each value of $\alpha$. to be unlikely since $\alpha>0.205\left(=\alpha_{100}\right)$ cannot be satisfied. The graph clearly shows that, as the electronic structure becomes more atomiclike (as indicated by the SCS's), the screening charge takes on more $s$ character, from $\sim 35 \%$ for bulk $\mathrm{Ta}$ to a maximum of $\sim 60 \%$ for $\mathrm{Ta}(100)$. Although one must be careful to keep in mind that the orbital character of the screening charge is not strictly equivalent to the orbital character of the conduction band (since one describes the local charge surrounding a point defect and the other the delocalized charge in the surface layer or bulk), a close correspondence is expected. Thus, in a fairly direct manner, the singularity-index results show an increase in $s$-electron charge corresponding to the increase in atomic character of the Ta atoms.

The bulk lifetime width of $37 \pm 5 \mathrm{meV}$ is consistent with $40 \pm 10 \mathrm{meV}$ (Ref. 8) obtained from data on $\mathrm{Ta}(111)$ but slightly smaller than the $52 \pm 7$ (Ref. 4) and $50 \mathrm{meV}$ (Ref. 9) deduced from data on $\mathrm{Ta}(110)$. In these earlier studies the same lifetime broadening was assumed for the bulk and surface lines. The slightly larger values from $\mathrm{Ta}(110)$, which are surely averages of bulk and surface values, suggest that the surface lifetime width is also larger for $\mathrm{Ta}(110)$; however, the surface component is not well enough resolved to determine reliably a separate value for the broadening. The large uncertainty in the $\mathrm{Ta}(111)$ width precludes any assessment of a difference in surface lifetime for $\mathrm{Ta}(111)$.

The increase in the lifetime width to $70 \pm 20 \mathrm{meV}$ at the $\mathrm{Ta}(100)$ surface can be explained by the same mechanism used to account for an analogous observation on W(110). ${ }^{3}$ For W(110) the increased width was taken as an indication that the increased localization of the more atomiclike $d$-electron charge density increases the intra-atomic Auger core-valence-valence cross section. For $\mathrm{Ta}(100)$, interatomic Auger decay may also contribute to the increased width for the surface atoms: results from an Auger-photoemission coincidence study point to a $40 \%$ increase in the surface lifetime width from interatomic decay involving second-layer atoms. ${ }^{20}$ This result is entirely consistent with our present measurement.

Perhaps the largest surprise of the line-shape analysis comes from the Gaussian widths. If the Gaussian broadening is due only to instrumental and phonon effects (with no static inhomogeneity) then the deduced phonon widths are $114 \pm 14$ and $82 \pm 32 \mathrm{meV}$ for the bulk and surface, respectively. The bulk width is larger, but consistent with, a previous value of $88 \pm 20 \mathrm{meV},{ }^{4}$ also measured at $80 \mathrm{~K}$. The slightly larger value measured here suggests that the bulk component in the $\mathrm{Ta}(100)$ data may be inhomogeneously broadened due to slightly different binding energies for atoms in several layers below the first two, although analyses with more underlayer components were generally worse than with just the three lines (bulk, underlayer, and surface). The smaller surface width implies a smaller phonon broadening for the first layer. Upon first consideration this is not expected since vibrations normal to the surface are characterized by a smaller Debye temperature $\Theta_{D}$ than in-plane vibrations (which are similar to vibrations in the bulk). ${ }^{21}$ In the alkali metals, for example, the surface phonon broadening is larger than for the bulk. ${ }^{22}$ However, the 
phonon broadening $G_{\text {el-ph }}$ depends not only on the effective Debye temperature but also on a coupling constant $C$ through the equation ${ }^{23,24}$

$$
G_{\text {el-ph }}^{2}=\frac{C}{\Theta_{D}}\left[1+\left(\frac{8}{3} \frac{T}{\Theta_{D}}\right)^{2}\right]^{1 / 2},
$$

where $T$ is the temperature. Since the constant $C$ depends upon the valence-band structure, ${ }^{24}$ it need not be the same at the surface and in the bulk. As far as we know this is the first experimental indication that the parameter $C$ may be altered within the surface layer of a metal. However, data over a wide temperature range including points well below $\Theta_{D}$ are required to separately extract values of $C$ and $\Theta_{D}$ from measurements of the Gaussian broadening.

The line-shape, SCS, and UCS results for $\mathrm{Ta}(100)$ resolve the discrepancy in interpretation between two earlier core-level studies of this surface. ${ }^{5,6}$ Our results show that the first study ${ }^{5}$ was qualitatively correct: The surface-atom line shape is characterized by a larger value of $\alpha$ and an enhanced lifetime width. The discrepancy in quantitative results between the first study and the present one comes from the neglect of any phonon broadening in the first study. Such neglect resulted in fitted values of $\Gamma_{B}=120 \pm 10 \mathrm{meV}, \Gamma_{100}=220 \pm 10 \mathrm{meV}$, $\alpha_{B}=0.08 \pm 0.02$, and $\alpha_{100}=0.18 \pm 0.02$. Compared to our present values, the larger Lorentzian widths simply arose in order to account for the disregarded phonon broadening. The smaller $\alpha$ 's were a secondary consequence caused by the longer tails of the too-large Lorentzian widths. The very small values of $\alpha=0.05$ reported in the second study ${ }^{6}$ were the result of attempting to fit the high-binding-energy side of the surface peak with a nonstandand screening-response feature which was postulated to arise from the bulk band structure. ${ }^{25}$ However, it has been previously shown that no such peak exists ${ }^{4}$ so that its invocation to explain the $\mathrm{Ta}(100)$-surface line shape is invalid. The present results for the bindingenergy shifts, $\mathrm{SCS}=718 \pm 5 \mathrm{meV}$ and $\mathrm{UCS}=125 \pm 5 \mathrm{meV}$, are also in reasonable agreement with the first study ${ }^{5}$ which deduced values of $740 \pm 10$ and $140 \pm 10 \mathrm{meV}$, respectively, but in strong disagreement with the second study, ${ }^{6}$ which showed spectra fitted with values close to 805 and $180 \mathrm{meV}$, respectively.

In summary, we have presented core-level photoemission data from (100)-oriented Ta metal which have yielded independent values of the singularity index and lifetime widths for surface and bulk atoms. We have further extracted surface singularity-index values for $\mathrm{Ta}(111)$ and $\mathrm{Ta}(110)$ and have related the exponents to the degree of $s$-wave screening of the core hole. The singularity indices obtained here and in earlier work on W(110) (Ref. 3) are consistent with the more atomiclike nature of the surface atoms, and suggest a trend for other $d$-band transition metals whose $d$-band charge is typically greater in the metallic state than in the free atom.

\section{ACKNOWLEDGMENTS}

This work was supported by NSF Grant No. DMR9303091 and the Robert A. Welch Foundation (University of Texas). The National Synchrotron Source is supported by the DOE Division of Material Science and the Division of Chemical Sciences.
${ }^{*}$ Present address: P.L.S., Pohang University of Science and Technology, Pohang, Republic of Korea.

${ }^{1}$ D. Spanjaard, C. Guillot, M. C. Desjonquères, G. Tréglia, and J. Lecante, Surf. Sci. Rep. 5, 1 (1985), and references therein.

${ }^{2}$ K. G. Purcell, J. Jupille, G. P. Derby, and D. A. King, Phys. Rev. B 36, 1288 (1987); G. K. Wertheim and P. H. Citrin, ibid. 38, 7872 (1988); J. Jupille, K. G. Purcell, and D. A. King, ibid. 39, 6871 (1989); D. M. Riffe, G. K. Wertheim, and P. H. Citrin, Phys. Rev. Lett. 65, 219 (1990); P. Alnot, D. J. Auerbach, J. Behm, and C. R. Brundle, Surf. Sci. 213, 1 (1989); C. H. F. Peden and N. D. Shinn, ibid. 312, 151 (1994).

${ }^{3}$ D. M. Riffe, G. K. Wertheim, and P. H. Citrin, Phys. Rev. Lett. 63, 1976 (1989).

${ }^{4}$ D. M. Riffe and G. K. Wertheim, Phys. Rev. B 47, 6672 (1993).

${ }^{5}$ C. Guillot, P. Roubin, J. Lecante, M. C. Desjonquères, G. Tréglia, D. Spanjaard, and Y. Jugnet, Phys. Rev. B 30, 5487 (1984).

${ }^{6}$ D. Sébilleau, C. Guillot, D. Chaveau, B. Villette, J. Lecante, M. C. Desjonquères, D. Spanjaard, and G. Tréglia, J. Phys. C 21, 287 (1988).

${ }^{7}$ J. F. van der Veen, P. Heimann, F. J. Himpsel, and D. E. Eastman, Solid State Commun. 37, 555 (1981); J. F. van der Veen, F. J. Himpsel, and D. E. Eastman, Phys. Rev. B 25, 7388 (1982).
${ }^{8}$ G. K. Wertheim, P. H. Citrin, and J. F. van der Veen, Phys. Rev. 30, 4343 (1984).

${ }^{9}$ C. Gu, D. W. Lynch, A. B. Yang, and C. G. Olson, Phys. Rev. B 42, 1526 (1990).

${ }^{10}$ S. Doniach and M. Šunjić, J. Phys. C 3, 285 (1970).

${ }^{11}$ The surface core-level shift is defined as the surface-atom core-electron binding energy minus the bulk-atom coreelectron binding energy (of a particular core level).

${ }^{12}$ R. G. Musket, W. McLean, C. A. Colmenares, D. M. Makowiecki, and W. J. Siekhaus, Appl. Surf. Sci. 10, 143 (1982).

${ }^{13}$ The $j=\frac{5}{2}$ components are inherently broader due to a Coster-Kronig decay process which does not exist for the $j=\frac{7}{2}$ components.

${ }^{14}$ Inhomogeneous broadening is broadening caused by slight disorder in the solid which results in slightly different chemical environments (and hence slightly different core-level binding energies) for nominally identical atoms.

${ }^{15}$ R. A. Bartynski et al., Phys. Rev. B 40, 5340 (1989).

${ }^{16}$ W. F. Egelhoff, Surf. Sci. Rep. 6, 253 (1987), and references therein.

${ }^{17}$ P. H. Citrin, G. K. Wertheim, and Y. Baer, Phys. Rev. B 16, 4256 (1977), and references therein. 
18 J. Friedel, Comments Solid State Phys. 2, 21 (1969).

${ }^{19}$ A. J. Freeman (unpublished).

${ }^{20}$ E. Jensen, R. A. Bartynski, M. Weinert, S. Hulbert, E. D. Johnson, and R. F. Garrett, Phys. Rev. B 41, 12468 (1990).

${ }^{21}$ D. P. Jackson, Surf. Sci. 43, 431 (1974).

${ }^{22}$ D. M. Riffe, G. K. Wertheim, and P. H. Citrin, Phys. Rev. Lett. 67, 116 (1991); G. K. Wertheim, D. M. Riffe, and P. H.
Citrin, Phys. Rev. B 49, 2277 (1994).

${ }^{23}$ A. H. Overhauser, as reported by L. G. Parratt, Rev. Mod. Phys. 31, 616 (1959); A. J. McAlister, Phys. Rev. 186, 595 (1969).

${ }^{24}$ L. Hedin and A. Rosengren, J. Phys. F 7, 1339 (1977).

${ }^{25}$ D. Sébilleau, G. Tréglia, M. C. Desjonquères, C. Guillot, D. Chaveau, and D. Spanjaard, J. Phys. C 20, 2647 (1987). 